\title{
Classification of Intervertebral Disc Degeneration in Low Back Pain Using Diffusional Kurtosis Imaging
}

\author{
Hiromitsu Takano,,2, Ikuho Yonezawa ${ }^{2,3}$, Takatoshi Okuda², Kazuo Kaneko² \\ ${ }^{1}$ Department of Orthopedic Surgery, Koto Hospital, Tokyo, Japan \\ ${ }^{2}$ Department of Orthopedic Surgery, School of Medicine, Juntendo University, Tokyo, Japan \\ ${ }^{3}$ Department of Spine Surgery, Sangubashi Spine Surgery Hospital, Tokyo, Japan \\ Email: hrtakano@juntendo.ac.jp
}

How to cite this paper: Takano, H., Yonezawa, I., Okuda, T. and Kaneko, K. (2020) Classification of Intervertebral Disc Degeneration in Low Back Pain Using Diffusional Kurtosis Imaging. Open Journal of Radiology, 10, 79-89.

https://doi.org/10.4236/ojrad.2020.102009

Received: May 11, 2020

Accepted: June 7, 2020

Published: June 10, 2020

Copyright $\odot 2020$ by author(s) and Scientific Research Publishing Inc. This work is licensed under the Creative Commons Attribution International License (CC BY 4.0).

http://creativecommons.org/licenses/by/4.0/

\begin{abstract}
Degenerative disc disease is the most common cause of low back pain. Intervertebral disc abnormalities are commonly evaluated by magnetic resonance imaging (MRI), and Pfirmann's system involves the use of T2-weighted images (T2WI) to classify disc degeneration. However, as this classification is based on visual evaluation, it is not possible to quantify degeneration using this method. The present study was performed to establish an MRI-based intervertebral disc classification system using diffusional kurtosis imaging (DKI), to quantify intervertebral disc water content according to the Pfirrmann classification. Sagittal mean diffusional kurtosis (MK) mapping was performed for the $\mathrm{L} 3 / 4, \mathrm{~L} 4 / 5$, and L5/S1 intervertebral discs in 32 patients (15 female, 17 male; age range, 24 - 82 years; mean age, 57.7 years). The degree of disc degeneration was assessed in the midsagittal section on T2WI according to the Pfirrmann classification (grade I - V). The relationships between MK values, which are correlated with intervertebral disc composition changes, and grade of degeneration determined using the Pfirrmann classification were analyzed. The MK values tended to decrease with increasing grade of degeneration, and differed significantly between grades I and IV, but not between grade IV and V $(\mathrm{P}<0.05$, Mann-Whitney $\mathrm{U}$ test $)$. DKI is an effective means of detecting the early stages of disc degeneration. Therefore, DKI may be a useful diagnostic tool for quantitative assessment of intervertebral disc degeneration.
\end{abstract}

\section{Keywords}

Diffusional Kurtosis Imaging, Pfirrmann Classification, Mean Diffusional Kurtosis, Intervertebral Disc Degeneration, Low Back Pain 


\section{Introduction}

Low back pain is one of the most common causes of disability in the working-age population, and has a number of known causes [1] [2], the most common of which is degenerative disc disease [3] [4]. Magnetic resonance imaging (MRI) is a useful noninvasive tool for clinical assessment of intervertebral disc pathology, and T2-weighted images (T2WI) reflect changes in discs due to aging or degeneration, and allow determination of disc degeneration. The normal disc has a central portion of high signal intensity and a peripheral portion of decreased signal intensity [5] [6] [7]. Changes in MRI signal strength in the nucleus pulposus can indicate disc degeneration. MRI is a useful tool to quantify degenerative intervertebral discs [8] [9]. Pfirrmann et al. developed a system for classifying the degree of disc degeneration based on T2WI findings [10] [11] [12]. However, as this classification is based on visual evaluation, it is not possible to quantify the degree of degeneration using this method. Mean diffusional kurtosis (MK) mapping is an MRI technology that allows quantification of water content, and can be used for the early detection of abnormalities in the cartilage as well as to track the responses to therapy. As the MK value is a quantitative parameter that varies with collagen and water contents in cartilage and intervertebral discs, it may be useful for characterizing the etiology of lower back pain and disc degeneration [13]. However, previous studies regarding the correlation between MK value and disc degeneration did not investigate the classification boundaries based on quantitative evaluation. A quantifiable method for classification of disc degeneration may be useful for research regarding disc abnormalities. The present study was performed to establish an intervertebral disc MRI classification system using diffusional kurtosis imaging (DKI), with an emphasis on the evaluation of early intervertebral disc degeneration, by quantifying disc water contents according to the Pfirrmann classification.

\section{Materials and Methods}

\subsection{Participants}

All patients provided written, informed consent prior to enrollment, and the study protocol was approved by the Ethics Committee of Juntendo University. A total of 32 patients (female, 15; male, 17) 24 - 82 years old (mean age, 57.7 years, standard deviation $[\mathrm{SD}] \pm 17.8$ ) with single or recurrent episodes of low back pain and leg numbness and tingling, including pain, were examined using an Achieva whole-body 3.0 T MR scanner (Philips Medical Systems, Best, The Netherlands) with a phased array spine coil (Table 1). Subjects were excluded if

Table 1. Demographic characteristics of the subjects.

\begin{tabular}{cc}
\hline Sex (male:female) & $15: 17$ \\
Mean Age (SD) & $57.7(17.8)$ \\
Symptoms (low back pain) & 32 \\
\hline
\end{tabular}


they had other intraspinal diseases, such as tumors, a history of lumbar surgery for any disease, or if image quality was unsatisfactory for diffusion metrics calculation [14] [15].

\subsection{Image Acquisition}

Images were acquired on an Achieva 3.0 T MR scanner (Philips Medical Systems) with the following imaging parameters for DKI: repetition time/echo time, 10,758/88 ms; number of excitations, two; slice thickness/gap, 4/0 mm; number of slices, 32; field of view, $64 \times 64 \mathrm{~mm}$; matrix, $128 \times 128$ reconstructed; imaging time, approximately $13 \mathrm{~min}$; and four b-values $\left(0,700,1400\right.$, and $2100 \mathrm{~s} / \mathrm{mm}^{2}$ ) with diffusion encoding in six directions for each $b$-value. The gradient length ( $\delta$ ) was $9.8 \mathrm{~ms}$ and the time between the two leading edges of the diffusion gradient $(\Delta)$ was $44.1 \mathrm{~ms}$. Image quality was improved by using a reduced field-of-view technique [14] [16] [17]. Prior to DKI, conventional turbo spin echo T1- and T2-weighted sagittal and axial images were obtained. For sagittal images, the imaging parameters were: repetition time/echo time, 400/10 ms for T1WI and 3246/128 ms for T2WI; echo train length, 4 for T1WI and 36 for T2WI; number of excitations, two; slice thickness/gap, $3 / 0.3 \mathrm{~mm}$; number of slices, 11 ; field of view, $250 \times 250 \mathrm{~mm}$; and matrix, $512 \times 512$. For axial images, the following imaging parameters were used: repetition time/echo time, 726/10 ms for T1WI and 6196/93 ms for T2WI; echo train length, 5 for T1WI and 36 for T2WI; number of excitations, two; slice thickness/gap, 4/0.4 mm; number of slices, 24; field of view, $160 \times 160 \mathrm{~mm}$; and matrix, $512 \times 512$.

\subsection{Analyses of DKI}

The free software dTV II FZRx and Volume-One 1.72 (Image Computing and Analysis Laboratory, Department of Radiology, The University of Tokyo Hospital, Tokyo, Japan) [18] were used for analyses of DKI on a PC running Windows (Microsoft, Redmond, WA). Disc degeneration was classified as grade I -V on the Pfirrmann classification in the midsagittal section on T2WI in a blinded manner (Figure 1 and Table 2). The MK map was generated using the MK values in the midsagittal section from sagittal sections centered on the lumbar midline region. The mean values in the regions of interest (ROI) were measured in the middle of five equal areas on three slices of the midsagittal section (Figure 2). Analyses were performed for each Pfirrmann grade to determine the MK values in the nucleus pulposus region [19].

\subsection{Statistical Analysis}

The Mann-Whitney U test was used for statistical analyses with IBM SPSS Statistics version 23.0. In all analyses, $\mathrm{P}<0.05$ was taken to indicate statistical significance.
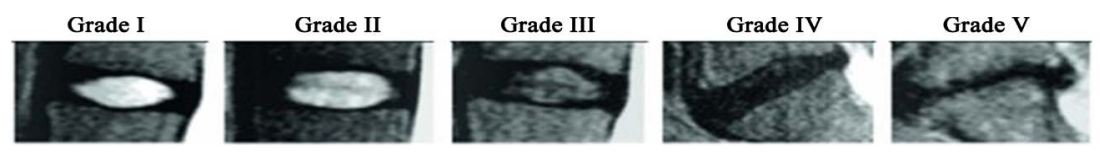

Figure 1. Pfirrmann classification [11] [12]. 
Table 2. Classification of intervertebral disc degeneration as reported by Pfirrmann et al. [13] [20].

\begin{tabular}{cccc}
\hline Grade Structure & $\begin{array}{c}\text { Distinction of the } \\
\text { nucleus and annulus }\end{array}$ & Signal intensity & $\begin{array}{c}\text { Height of the } \\
\text { intervertebral disk }\end{array}$ \\
\hline $\begin{array}{c}\text { I: Homogeneous, bright white } \\
\text { II: Heterogeneous with or without } \\
\text { horizontal bands }\end{array}$ & Clear & Hyperintense, isointense to CSF & Normal \\
III: Heterogeneous, gray & Unclear & Hyperintense, isointense to CSF & Normal \\
IV: Heterogeneous, gray or black & Lost & Intermediate or hypointense & Normal to moderately decreased \\
V: Heterogeneous, black & Lost & Hypointense & Collapsed disk space \\
\hline
\end{tabular}

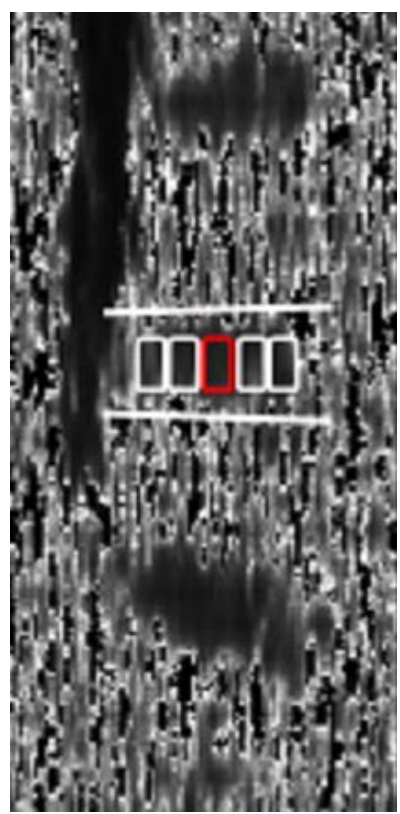

Figure 2. Measurement of the intervertebral disc. Discs were divided into five equal areas, and we measured the middle fifth of the nucleus pulposus midsagittal section on the MK map. The mean values were measured in the same ROI as in areas.

\section{Results}

Sagittal MK mapping was performed for the L3/4, L4/5, and L5/S1 intervertebral discs in 32 patients, total 96 discs. The distribution of T2WI-based Pfirrmann grade classification was as follows: grade I, 6 discs; grade II, 18 discs; grade III, 25 discs; grade IV, 32 discs; and grade V, 15 discs. DKI data with good image quality could not be obtained in some discs. The results of DKI analyses of successfully imaged discs were as follows: grade I, 4 discs; grade II, 10 discs; grade III, 16 discs; grade IV, 15 discs; and grade V, 1 disc. Detection rates of DKI were as follows: grade I, 66.7\%; grade II, 55.6\%; grade III, 64\%; grade IV, $46.9 \%$; and grade $\mathrm{V}, 6.7 \%$ (Table 3 ). The MK values measured in each grade in the nucleus pulposus are shown in Figure 3. The MK values (mean \pm standard deviation) for discs were as follows: grade I, $1.994 \pm 0.433$; grade II, $1.413 \pm 0.249$; grade III, $1.19 \pm 0.255$; grade IV, $1.848 \pm 0.735$; and grade V, 1.743 (Table 4). MK values 
Table 3. Detection rate of DKI: grade I, $66.7 \%$; grade II, $55.6 \%$; grade III, $64 \%$; grade IV, $46.9 \%$; grade V, $6.7 \%$; and total $47.9 \%$.

\begin{tabular}{ccccccc}
\hline Pfirrmann classification & Grade I & Grade II & Grade III & Grade IV & Grade V & Total \\
\hline Lumbar discs & 6 & 18 & 25 & 32 & 15 & 96 \\
MK map discs & 4 & 10 & 16 & 15 & 1 & 46 \\
Detection rate (\%) & 66.7 & 55.6 & 64 & 46.9 & 6.7 & 47.9 \\
\hline
\end{tabular}

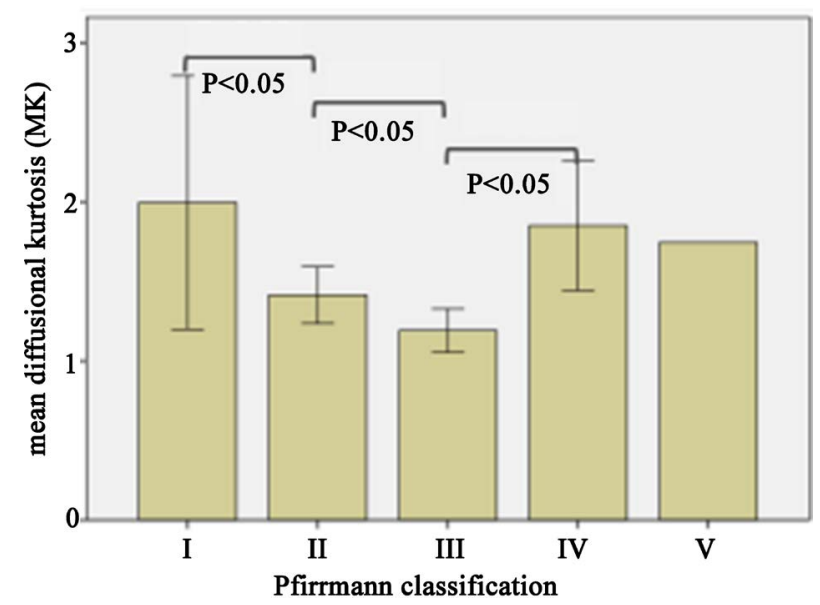

Figure 3. Relationship between Pfirrmann classification grade and MK value. In the nucleus pulposus, $\mathrm{MK}$ values tended to decrease with increasing grade, and there were significant differences from grades I to IV, but not between grades IV and V.

Table 4. MK values tended to decrease with increasing grade, and MK values were significantly different from grades I to IV, but not between grades IV and V.

\begin{tabular}{cccccc}
\hline Pfirrmann classification & Grade I & Grade II & Grade III & Grade IV & Grade V \\
\hline MK map discs & 4 & 10 & 16 & 15 & 1 \\
MK values & 1.994 & 1.413 & 1.19 & 1.848 & 1.743 \\
\pm SD & 0.433 & 0.249 & 0.255 & 0.735 & \\
P-value & & 0.024 & 0.027 & 0.002 & \\
\hline
\end{tabular}

tended to decrease with increasing grade, and were significantly different from grades I to IV, but not between grades IV and V $(\mathrm{P}<0.05$, Mann-Whitney U test).

\section{Discussion}

\subsection{Intervertebral Disc Degeneration}

A highly organized framework of collagen fibrils, representing the main macromolecular component of the intervertebral disc [20], anchors the disc to the bone and provides tensile strength. The highest levels of collagen are seen in the outer annulus of the disc (as much as $70 \%$ by dry weight), which decreases to approximately $20 \%-30 \%$ by dry weight in the nucleus of the adult human lumbar disc [21]. 
Proteolysis of matrix macromolecules plays a role in intervertebral disc degeneration, with the proteolytic products slowly diffusing out of the disc leading to a loss of matrix integrity, failure of biomechanical load response, and ultimately to the morphological features associated with degeneration. The sequential changes that occur in this process are still not well understood, but the earliest and most marked degenerative change in disc composition is the loss of glycosaminoglycan (GAG) [22], which decreases in parallel with increasing grade of disc degeneration. The loss of GAG results in a drop in swelling pressure [23], loss of hydration, and loss of disc height, with resulting adverse effects on the ability of the disc to respond appropriately to applied biomechanical loads. Disc degeneration also results in disorganization and destruction of the collagen network [24], and is generally classified based on T2WI findings using the system described by Pfirrmann et al. [10] [11] [12]. However, this classification is based on visual evaluation, and therefore it cannot be used to quantify the degree of degeneration. The water and proteoglycan contents within tissues can be quantified by magnetic resonance T2 mapping, and this method can be used to detect early cartilage abnormalities as well as to track responses to therapy [19]. The T2 value tended to decrease with increasing Pfirmann classification grade, and $\mathrm{T} 2$ values differed significantly from grades I to IV, but not grades IV and $\mathrm{V}[5]$.

\subsection{Diffusional Kurtosis Imaging (DKI)}

Diffusion-weighted imaging (DWI) [25], including diffusion tensor imaging (DTI) [26], is based on the Gaussian distribution and random movement of water molecules, while DKI [27] visualizes non-Gaussian water diffusion, and has been applied clinically for the characterization of normal and abnormal tissues. In contrast to the mature metrics of DTI, such as fractional anisotropy (FA), DKI and its derived parameters are currently still in the developmental stage [28] [29]. However, a number of reports have indicated important features of DKI for clinical applications, such as its potential usefulness in diagnostic imaging for the evaluation of neurological issues associated with aging [30] as well as various disorders, such as stroke, Alzheimer's disease, schizophrenia, glioma [31] [32], Parkinson's disease [33], and attention deficit hyperactivity disorder. There have also been trials to examine the applicability of DKI in the liver [34] and spine [18], and DKI may facilitate estimation of conventional DTI parameters with improved accuracy [35].

\subsection{Classification of Intervertebral Disc Degeneration Based on MK Values}

Intervertebral disc degeneration is commonly classified according to the method of Pfirrmann et al. [11], which involves determination of the amount of water in the disc based on the T2WI signal intensity, as the water content decreases with both age and disc degeneration [36]. Although T2WI is used clinically for evalu- 
ation of disc degeneration [37] [38] [39], it is not possible to determine the absolute signal intensity [40]. In the present study, we performed MK mapping to determine the MK value of the water content in intervertebral discs according to the Pfirrmann classification. Our results indicated that the MK values tended to decrease with increasing Pfirrmann classification grade in the nucleus pulposus, which may reflect reductions of both proteoglycan and water contents [8] [9]. However, there was no significant difference in MK value between grade IV and $\mathrm{V}$ of the Pfirrmann classification, and therefore it may be difficult to distinguish between these two grades based on the MK values. In most previous studies, the ROI was selected manually, which has low reproducibility due to the poor differentiation of the central nuclear region from the peripheral annulus. In the present study, the ROI was selected by measuring small equally divided areas. This technique has improved reproducibility compared to the manual approach as well as minimal measurement bias. In the present study, the MK values were shown to decrease with increasing Pfirrmann classification grade in the nucleus pulposus, which probably reflected decreases in both proteoglycan and water contents. Grade IV and V of the Pfirrmann classification increased MK values, since the spatial resolution was not sufficient, it would be difficult to draw an ROI on grade IV and V. Here, we proposed boundaries of the classification based on quantitative evaluation, and therefore our findings suggested that determination of intervertebral disc water content based on the MK value may be useful in clinical research regarding degenerative disc diseases.

\section{Conclusions}

- The present study was performed to establish an MRI-based classification system for intervertebral disc degeneration using DKI.

- Our results indicate that DKI was effective for detecting the early stages of disc degeneration.

- DKI may be useful as a diagnostic tool for quantitative assessment of intervertebral disc degeneration.

- The use of MRI represents an effective objective rating system for studies of new surgical techniques or for drug-based therapies to prevent intervertebral disc degeneration.

\section{Acknowledgements}

All authors contributed to proofreading and editing the manuscript before submission.

\section{Ethics Approval and Consent to Participate}

We obtained the informed consent from the patient to report this case.

\section{Human and Animal Rights}

No animals/humans were used for studies that are base of this research. 


\section{Conflicts of Interest}

The authors declare no conflicts of interest regarding the publication of this paper.

\section{References}

[1] Andersson, G.B. (1998) Epidemiology of Low Back Pain. Acta Orthopaedica Scandinavica Supplementum, 281, 28-31. https://doi.org/10.1080/17453674.1998.11744790

[2] Benneker, L.M., Heini, P.F., Anderson, S.E., Alini, M. and Ito, K. (2005) Correlation of Radiographic and MRI Parameters to Morphological and Biochemical Assessment of Intervertebral Disc Degeneration. European Spine Journal, 14, 27-35. https://doi.org/10.1007/s00586-004-0759-4

[3] Paajanen, H., Erkintalo, M., Parkkola, R., Salminen, J. and Kormano, M. (1997) Age-Dependent Correlation of Low-Back Pain and Lumbar Disc Regeneration. Archives of Orthopaedic and Trauma Surgery, 116, 106-107. https://doi.org/10.1007/BF00434112

[4] Salminen, J.J., Erkintalo, M.O., Pentti, J., Oksanen, A. and Kormano, M.J. (1999) Recurrent Low Back Pain and Early Disc Degeneration in the Young. Spine, 24, 1316-1321. https://doi.org/10.1097/00007632-199907010-00008

[5] Modic, M.T., Masaryk, T.J., Ross, J.S. and Carter, J.R. (1988) Imaging of Degenerative Disk Disease. Radiology, 168, 177-186.

https://doi.org/10.1148/radiology.168.1.3289089

[6] Pearce, R.H., Thompson, J.P., Bebault, G.M. and Flak, B. (1991) Magnetic Resonance Imaging Reflects the Chemical Changes of Aging Degeneration in the Human Intervertebral Disk. The Journal of Rheumatology, 27, 42-43.

[7] Sether, L.A., Yu, S., Haughton, V.M. and Fischer, M.E. (1990) Intervertebral Disk: Normal Age-Related Changes in MR Signal Intensity. Radiology, 177, 385-388. https://doi.org/10.1148/radiology.177.2.2217773

[8] Urban, J.P. and McMullin, J.F. (1988) Swelling Pressure of the Lumbar Intervertebral Discs: Influence of Age, Spinal Level, Composition, and Degeneration. Spine, 3, 179-187. https://doi.org/10.1097/00007632-198802000-00009

[9] Zou, J., Yang, H., Miyazaki, M., Morishita, Y., Wei, F., McGovern, S. and Wang, J.C. (2009) Dynamic Bulging of Intervertebral Discs in the Degenerative Lumbar Spine. Spine, 34, 2545-2550. https://doi.org/10.1097/BRS.0b013e3181b32998

[10] Schneiderman, G., Flannigan, B., Kingston, S., Thomas, J., Dillin, W.H. and Watkins, R.G. (1987) Magnetic Resonance Imaging in the Diagnosis of Disc Degeneration: Correlation with Discography. Spine, 12, 276-281. https://doi.org/10.1097/00007632-198704000-00016

[11] Pfirrmann, C., Metzdorf, A., Zanetti, M., Hodler, J. and Boos, N. (2001) Magnetic Resonance Classification of Lumbar Intervertebral Disc Degeneration. Spine, 26, 1873-1878. https://doi.org/10.1097/00007632-200109010-00011

[12] Hangai, M., Kaneoka, K., Kuno, S., Hinotsu, S., Sakane, M., Mamizuka, N., Sakai, S. and Ochiai, N. (2008) Factors Associated with Lumbar Intervertebral Disc Degeneration in the Elderly. The Spine Journal, 8, 732-740. https://doi.org/10.1016/j.spinee.2007.07.392

[13] Boos, N., Wallin, A., Gbedegbegnon, T., Aebi, M. and Boesch, C. (1993) Quantitative MR Imaging of Lumbar Intervertebral Disks and Vertebral Bodies: Influence of Diurnal Water Content Variations. Radiology, 188, 351-354. 
https://doi.org/10.1148/radiology.188.2.8327677

[14] Hori, M., Tsutsumi, S., Yasumoto, Y., Ito, M., Suzuki, M., Tanaka, F.S., Kyogoku, S., Nakamura, M., Tabuchi, T., Fukunaga, I., Suzuki, Y., Kamagata, K., Masutani, Y. and Aoki, S. (2014) Cervical Spondylosis: Evaluation of Microstructural Changes in Spinal Cord White Matter and Gray Matter by Diffusional Kurtosis Imaging. Magnetic Resonance Imaging, 32, 428-432. https://doi.org/10.1016/j.mri.2014.01.018

[15] Trattnig, S., Stelzeneder, D., Goed, S., Reissegger, M., Mamisch, T.C., Paternostro-Sluga, T., Weber, M., Szomolanyi, P. and Welsch, G.H. (2010) Lumbar Intervertebral Disc Abnormalities: Comparison of Quantitative T2 Mapping with Conventional MR at 3.0 T. European Radiology, 20, 2715-2722.

https://doi.org/10.1007/s00330-010-1843-2

[16] Wilm, B.J., Svensson, J., Henning, A., Pruessmann, K.P., Boesiger, P. and Kollias, S.S. (2007) Reduced Field-of-View MRI Using Outer Volume Suppression for Spinal Cord Diffusion Imaging. Magnetic Resonance in Medicine, 57, 625-630. https://doi.org/10.1002/mrm.21167

[17] Wilm, B.J., Gamper, U., Henning, A., Pruessmann, K.P., Kollias, S.S. and Boesiger, P. (2009) Diffusion Weighted Imaging of the Entire Spinal Cord. NMR in Biomedicine, 22, 174-181. https://doi.org/10.1002/nbm.1298

[18] Hori, M., Fukunaga, I., Masutani, Y., Taoka, T., Kamagata, K., Suzuki, Y. and Aoki, S. (2012) Visualizing Non-Gaussian Diffusion: Clinical Application of q-Space Imaging and Diffusional Kurtosis Imaging of the Brain and Spine. Magnetic Resonance in Medical Sciences, 11, 221-233. https://doi.org/10.2463/mrms.11.221

[19] Takashima, H., Takebayashi, T., Yoshimoto, M., Terashima, Y., Tsuda, H., Ida, K. and Yamashita, T. (2012) Correlation between T2 Relaxation Time and Intervertebral Disk Degeneration. Skeletal Radiology, 41, 163-167. https://doi.org/10.1007/s00256-011-1144-0

[20] Eyre, D.R. and Muir, H. (1974) Collagen Polymorphism: Two Molecular Species in Pig Intervertebral Disc. FEBS Letters, 42, 192-196. https://doi.org/10.1016/0014-5793(74)80783-0

[21] Eyre, D.R. and Muir, H. (1977) Quantitative Analysis of Types I and II Collagens in Human Intervertebral Discs at Various Ages. Biochimica et Biophysica Acta, 492, 29-42. https://doi.org/10.1016/0005-2795(77)90211-2

[22] Urban, J.P. and McMullin, J.F. (1985) Swelling Pressure of the Intervertebral Disc: Influence of Proteoglycan and Collagen Contents. Biorheology, 22, 145-157. https://doi.org/10.3233/BIR-1985-22205

[23] Lyons, G., Eisenstein, S.M. and Sweet, M.B. (1981) Biochemical Changes in Intervertebral Disc Degeneration. Biochimica et Biophysica Acta, 673, 443-453. https://doi.org/10.1016/0304-4165(81)90476-1

[24] Roberts, S., Evans, H., Trivedi, J. and Menage, J. (2006) Histology and Pathology of the Human Intervertebral Disc. The Journal of Bone and Joint Surgery, 88, 10-14. https://doi.org/10.2106/00004623-200604002-00003

[25] Le Bihan, D., Breton, E., Lallemand, D., Grenier, P., Cabanis, E. and Laval-Jeantet, M. (1986) MR Imaging of Intravoxel Incoherent Motions: Application to Diffusion and Perfusion in Neurologic Disorders. Radiology, 161, 401-407. https://doi.org/10.1148/radiology.161.2.3763909

[26] Basser, P.J., Mattiello, J. and LeBihan, D. (1994) Estimation of the Effective SelfDiffusion Tensor from NMR Spin Echo. Journal of Magnetic Resonance, Series B, 103, 247-254. https://doi.org/10.1006/jmrb.1994.1037

[27] Jensen, J.H., Helpern, J.A., Ramani, A., Lu, H. and Kaczynski, K. (2005) Diffusional 
Kurtosis Imaging: The Quantification of Non-Gaussian Water Diffusion by Means of Magnetic Resonance Imaging. Magnetic Resonance in Medicine, 53, 1432-1440. https://doi.org/10.1002/mrm.20508

[28] Matsunami, Y. and Aoki, S. (2014) Fast and Robust Estimation Diffusional Kurtosis Imaging (DKI) Parameters by General Closed-Form Expressions and Their Extensions. Magnetic Resonance in Medical Sciences, 13, 97-115. https://doi.org/10.2463/mrms.2013-0084

[29] Katsura, M., Suzuki, Y., Hata, J., Hori, M., Sasaki, H., Akai, H., Mori, H., Kunimatsu, A., Masutani, Y., Aoki, S. and Ohtomo, K. (2014) Non-Gaussian DiffusionWeighted Imaging for Assessing Diurnal Changes in Intervertebral Disc Microstructure. The Journal of Magnetic Resonance, 40, 1208-1214. https://doi.org/10.1002/jmri.24459

[30] Falangola, M.F., Jensen, J.H., Babb, J.S., Hu, C., Castellanos, F.X., Di Martino, A., Ferris, S.H. and Helpern, J.A. (2008) Age-Related Non-Gaussian Diffusion Patterns in the Prefrontal Brain. The Journal of Magnetic Resonance, 28, 1345-1350. https://doi.org/10.1002/jmri.21604

[31] Raab, P., Hattingen, E., Franz, K., Zanella, F.E. and Lanfermann, H. (2010) Cerebral Gliomas: Diffusional Kurtosis Imaging Analysis of Microstructural Differences. Radiology, 254, 876-881. https://doi.org/10.1148/radiol.09090819

[32] Van Cauter, S., Veraart, J., Sijbers, J., Peeters, R.R., Himmelreich, U., De Keyzer, F., Van Gool, S.W., Van Calenbergh, F., De Vleeschouwer, S., Van Hecke, W. and Sunaert, S. (2012) Gliomas: Diffusion Kurtosis MR Imaging in Grading. Radiology, 263, 492-501. https://doi.org/10.1148/radiol.12110927

[33] Wang, J.J., Lin, W.Y., Lu, C.S., Weng, Y.H., Ng, S.H., Wang, C.H., Liu, H.L., Hsieh, R.H., Wan, Y.L. and Wai, Y.Y. (2011) Parkinson Disease: Diagnostic Utility of Diffusion Kurtosis Imaging. Radiology, 261, 210-217.

https://doi.org/10.1148/radiol.11102277

[34] Rosenkrantz, A.B., Sigmund, E.E., Winnick, A., Niver, B.E., Spieler, B., Morgan, G.R. and Hajdu, C.H. (2012) Assessment of Hepatocellular Carcinoma Using Apparent Diffusion Coefficient and Diffusion Kurtosis Indices: Preliminary Experience in Fresh Liver Explants. Magnetic Resonance Imaging, 30, 1534-1540. https://doi.org/10.1016/j.mri.2012.04.020

[35] Hui, E.S., Cheung, M.M., Qi, L. and Wu, E.X. (2008) Towards Better MR Characterization of Neural Tissue Using Directional Diffusion Kurtosis Analysis. Neuroimage, 42, 122-134. https://doi.org/10.1016/j.neuroimage.2008.04.237

[36] Krueger, E.C., Perry, J.O., Wu, Y. and Haughton, V.M. (2007) Changes in T2 Relaxation Times Associated with Maturation of the Human Intervertebral Disk. American Journal of Neuroradiology, 28, 1237-1241. https://doi.org/10.3174/ajnr.A0546

[37] Chiu, E.J., Newitt, D.C., Segal, M.R., Hu, S.S., Lotz, J.C. and Majumdar, S. (2001) Magnetic Resonance Imaging Measurement of Relaxation and Water Diffusion in the Human Lumbar Intervertebral Disc under Compression in Vitro. Spine, 26, E437-E444. https://doi.org/10.1097/00007632-200110010-00017

[38] Gundry, C.R. and Fritts, H.M. (1997) Magnetic Resonance Imaging of the Musculoskeletal System. VIII. The Spine, Section 2. Clinical Orthopaedics and Related Research, 343, 260-271. https://doi.org/10.1097/00003086-199710000-00038

[39] Modic, M.T., Pavlicek, W., Weinstein, M.A., Boumphrey, F., Ngo, F., Hardy, R. and Duchesneau, P.M. (1984) Magnetic Resonance Imaging of Intervertebral Disk Disease. Clinical and Pulse Sequence Considerations. Radiology, 152, 103-111. 
https://doi.org/10.1148/radiology.152.1.6729099

[40] Watanabe, A., Benneker, L.M., Boesch, C., Watanabe, T., Obata, T. and Anderson, S.E. (2007) Classification of Intervertebral Disk Degeneration with Axial T2 Mapping. American Journal of Roentgenology, 189, 936-942.

https://doi.org/10.2214/AJR.07.2142 